\title{
Swallowing Function in Patients who Underwent Hemiglossectomy and Reconstruction with a Pectoralis Major Myocutaneous Flap
}

\author{
Kazuhide MATSUNAGA ${ }^{1)}$, Kazunori MORI ${ }^{2}$, Shinichi ASAMURA ${ }^{1)}$, \\ Tetsuji NAGATA ${ }^{3)}$ and Noritaka ISOGAI ${ }^{1)}$ \\ ${ }^{1)}$ Department of Plastic and Reconstructive Surgery, Kinki University School of Medicine \\ ${ }^{2)}$ Department of Otorhinolaryngology, Kinki University School of Medicine \\ ${ }^{3)}$ Department of Oral and Maxillofacial Surgery, Hamamatsu University School of Medicine
}

\begin{abstract}
We evaluated swallowing function in four tongue cancer patients who underwent hemiglossectomy and reconstruction with the pectoralis major myocutaneous flap at 12 months postoperatively. The size of the pectoralis major myocutaneous flap was almost $12 \times 5 \mathrm{~cm}$ in those four patients. In all patients, the flaps decreased in comparison with the size one month postoperatively. We performed functional evaluation. All patients were capable of oral ingestion. Patients who underwent excision of less than $50 \%$ of the radix linguae could consume an approximately normal diet. However, patients who underwent excision of more than $50 \%$ of the radix linguae required strict dietary limitations. We evaluated video fluorography findings. None of the patients could hold the test diet in the oral cavity. All patients could shift the residual tongue to the flap side as a compensatory function and the residual tongue could make good contact with the hard palate. There was no aspiration in any patient. Widening of the esophageal entrance during swallowing appeared good in all patients. We suggest that swallowing function in patients who underwent hemiglossectomy and reconstruction with a pectoralis major myocutaneous flap was good approximately at 12 months postoperatively.
\end{abstract}

Key Words : swallowing function, hemiglossectomy, pectoralis major myocutaneous flap

\section{Introduction}

It is necessary for plastic and reconstructive surgeons to evaluate swallowing function in tongue cancer patients who have undergone glossectomy and reconstruction with flaps. Typical choices for reconstruction in patients undergoing hemiglossectomy are free foream flap, free rectus abdominal flap and pectoralis major myocutaneous flap. In our hospital, we

Correspondence to : Kazuhide Matsunaga

Department of Plastic and Reconstructive Surgery, Kinki University School of Medicine, 377-2 Onohigashi, Osaka-sayama, Osaka 589-8511, Japan have performed reconstruction using the pectoralis major myocutaneous flap immediately after hemiglossectomy in tongue cancer patients. In this study, we evaluated swallowing function 12 months postoperatively in four tongue cancer paitients who underwent hemiglossectomy and reconstruction with the pectoralis major myocutaneous flap.

\section{Patients and Methods}

\section{Patients}

Four (male : 3, female : 1) tongue cancer patients who underwent hemiglossectomy and reconstruction with the pectoralis major 
日本外科系連合学会誌 第 33 卷 6 号

Table 1 Surgical findings of hemiglossectomy in four patients

\begin{tabular}{|c|c|c|c|c|}
\hline case & 1 & 2 & 3 & 4 \\
\hline Age / sex & $72 /$ Male & 24 / Female & 65 / Male & 72 / Male \\
\hline Neck dissection & \multicolumn{4}{|c|}{ Bilateral: conservative } \\
\hline $\begin{array}{l}\text { Resection of suprahyoid } \\
\text { muscles (tumor side) }\end{array}$ & \multicolumn{4}{|c|}{$\begin{array}{l}\text { Stylohyoideus } \\
\text { Mylohyoideus } \\
\text { Geniohyoideus } \\
\text { Anterior and posterior bellies of the digastricus }\end{array}$} \\
\hline $\begin{array}{l}\text { Cricopharyngeal myotomy } \\
\text { (tumor side) }\end{array}$ & \multicolumn{4}{|c|}{ m 1} \\
\hline Hyoid bone suspension & \multicolumn{2}{|c|}{-} & \multicolumn{2}{|c|}{+} \\
\hline Size of PMMC-flap (cm) & $12 \times 6$ & $12 \times 5$ & $12 \times 5$ & $12 \times 5$ \\
\hline
\end{tabular}

- : no operation, +: operation
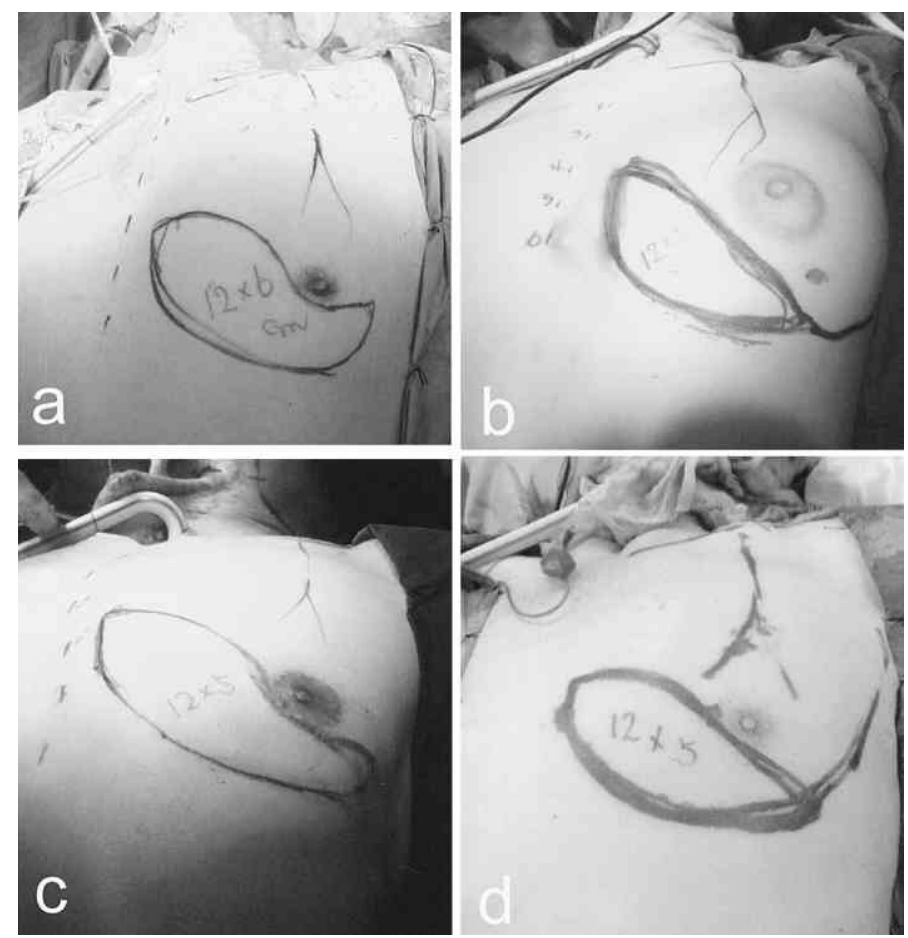

Fig. 1 Design of pectoralis major myocutaneous flap a : Case1, b: Case2, c: Case3, d: Case4

myocutaneous flap at Kinki University Hosipital, Osaka, Japan between January 2006 and December 2006 were studied. Patients ages ranged from 24 to 72 years old, with an average of 58.3 years old (Table $\mathbf{1}$ ).

Surgical technique
Table 1 shows the surgical technique. With regard to oral incision, all patients underwent hemiglossectomy including unilateral radix linguae and oral floor mucosa, but in all patients, the linguogingival mucosa was conserved. With regard to radical neck dissection, all 
Swallowing Function after Hemiglossectomy

Table 2 Postoperative treatment following hemiglossectomy in four patients

\begin{tabular}{c|c|c|c|c}
\hline Case & 1 & 2 & 3 & 4 \\
\hline pTNM & pT3N2bM0 & pT2N2bM0 & pT2N2bM0 & pT3N2bM0 \\
\hline Radiation & - & & $60 G y$ & \\
\hline
\end{tabular}

Table 3 Evaluation items (Functional evaluation)

\begin{tabular}{l|l}
\hline & \multicolumn{1}{|c}{ Item } \\
\hline 1 & Alimentation \\
\hline 2 & Quantity of ingestion (in comparison with that one month after surgery) \\
\hline 3 & Aspiration of water (subjective symptom) \\
\hline 4 & Staple diet / Side dishes \\
\hline
\end{tabular}

patients underwent bilateral conservative neck dissection. With regard to suprahyoid muscle dissection, all patients underwent unilateral (tumor side) excision of the stylohyoideus, mylohyoideus, geniohyoideus, anterior and posterior bellies of the digastricus muscles. Then, all patients underwent cricopharyngeal myotomy (tumor side). Furthermore, two patients (cases 3 and 4) who underwent excision of more than $50 \%$ of the radix linguae underwent hyoid bone suspension. The size of the pectoralis major myocutaneous flap of four patients was almost $12 \times 5 \mathrm{~cm}$ (Fig. 1). The flap was designed to include perforators of the 5 th and 6th posterior intercostal artery and vein $^{1)}$.

Postoperative treatment

In our hospital, patients with multiple regional metastases or extracapsular spread to the cervical lymph nodes are referred for postoperative radiation. three patients (cases 2,3 and 4 ) received 60 Gy radiation (Table 2 ). However, case 1 with multiple regional metastases refused postoperative radiation.

Evaluation

1) Serial changes in the flap

We visually evaluated changes in the flap at 12 months postoperatively in comparison with the size one month postoperatively.

2) Functional evaluation

Evaluation items were (1) alimentation, (2) quantity of ingestion in comparison with that one month after surgery, (3) aspiration of water (subjective symptom), (4) staple diet / side dishes (Table 3 ).

3) Video fluorography

Video fluorography was evaluated in three patients (cases. 1, 3, and 4). Video fluorography was performed with the patient's consent. The test diet consisted of $10 \mathrm{ml}$ liquid. Evaluation items were (1) holding the test diet in the oral cavity, (2) tongue movement, for example, contact between the hard plate and tongue, (3) penetration / aspiration, (4) the degree of maximal widening of the esophageal entrance (Table 4 ). The degree of maximal widening of the esophageal entrance during swallowing was examined using an image analysis system. With regard to quantitative analysis of the degree of maximal widening of the esophageal entrance, the longitudinal length of the three cervical vertebra on lateral images was established as 10.0. Then, we evaluated the degree of maximal widening of the esophageal entranc during swallowing of the test diet, as described by Matsunaga et $\mathrm{al}^{2,3)}$. 
日本外科系連合学会誌 第 33 卷 6 号

Table 4 Evaluation items (Video fluorography)

\begin{tabular}{c|l}
\hline & \multicolumn{1}{|c}{ Item } \\
\hline 1 & Holding test diet in the oral cavity \\
\hline 2 & Tongue movement (contact between the hard plate and tongue) \\
\hline 3 & Penetration / Aspiration \\
\hline 4 & The degree of maximal widening of the esophageal entrance \\
\hline \multicolumn{2}{c}{ cases: $1,3,4$} \\
& test food: the liquid $10 \mathrm{ml}$
\end{tabular}

Table 5 Functional evaluation (12 months postoperatively)

\begin{tabular}{|c|c|c|c|c|}
\hline Case & 1 & 2 & 3 & 4 \\
\hline Hyoid bone suspension & \multicolumn{2}{|c|}{-} & \multicolumn{2}{|c|}{+} \\
\hline Postoperative radiation & - & \multicolumn{3}{|c|}{$60 \mathrm{~Gy}$} \\
\hline Serial changes in flap & \multicolumn{2}{|c|}{ Decrease } & Remarkable decrease & Decrease \\
\hline 1 Alimentation & \multicolumn{4}{|c|}{ Oral ingestion } \\
\hline 2 Quantity of ingestion & No variation & Increase & Decrease & Increase \\
\hline 3 Aspiration of water & \multicolumn{4}{|c|}{ None (※) } \\
\hline 4 Staple diet / Side dish & $\begin{array}{l}\text { Well-boiled rice/ } \\
\text { soft diet }\end{array}$ & $\begin{array}{l}\text { Boiled rice/ } \\
\text { regular diet }\end{array}$ & Rice gruel/paste & Rice gruel/ paste \\
\hline
\end{tabular}

\section{Results}

1) Serial changes in the flap

Fig. 2 shows postoperative serial changes in the flaps in all four patients. Flaps of all patients decreased at 12 months postoperatively in comparison with the size one month postoperatively. Particularly, the flap in case 3 decreased remarkably and descended downward (Fig. 2 c- 2 ).

\section{2) Functional evaluation}

Table 5 shows the results of functional evaluation. With regard to alimentation, all patients could take oral ingestion. The quantity of ingestion varied among four patients. The quantities that could be ingested showed an increase in two patients. Aspiration of water (subjective symptom) was not observed in any patient. Two patients (cases 1 and 2 ) who underwent excision of less than $50 \%$ of the radix linguae could consume an almost normal diet. However, the other two patients (cases 3 and 4 ) who underwent excision of more than $50 \%$ of the radix linguae could consume only rice gruel and paste.

3) Video fluorography

None of the three patients examined could hold the test diet in the oral cavity, and flowing of the test diet into the pharynx was observed (Fig. 3). With regard to tongue movement on antero-posterior view, we observed that all patients could shift the residual tongue to the flap side as a compensatory function and the residual tongue could make good contact with the hard palate (Fig. 4 ). Penetration was observed in only one patient (case 4). However, there was no apparent aspiration in any patient (Fig. 5). Widening of the esophageal entrance during swallowing was good in all three patients. And the degree of maximal widening of the esophageal entrance during swallowing ranged from 10.3-11.8 (Fig. 6 ). 

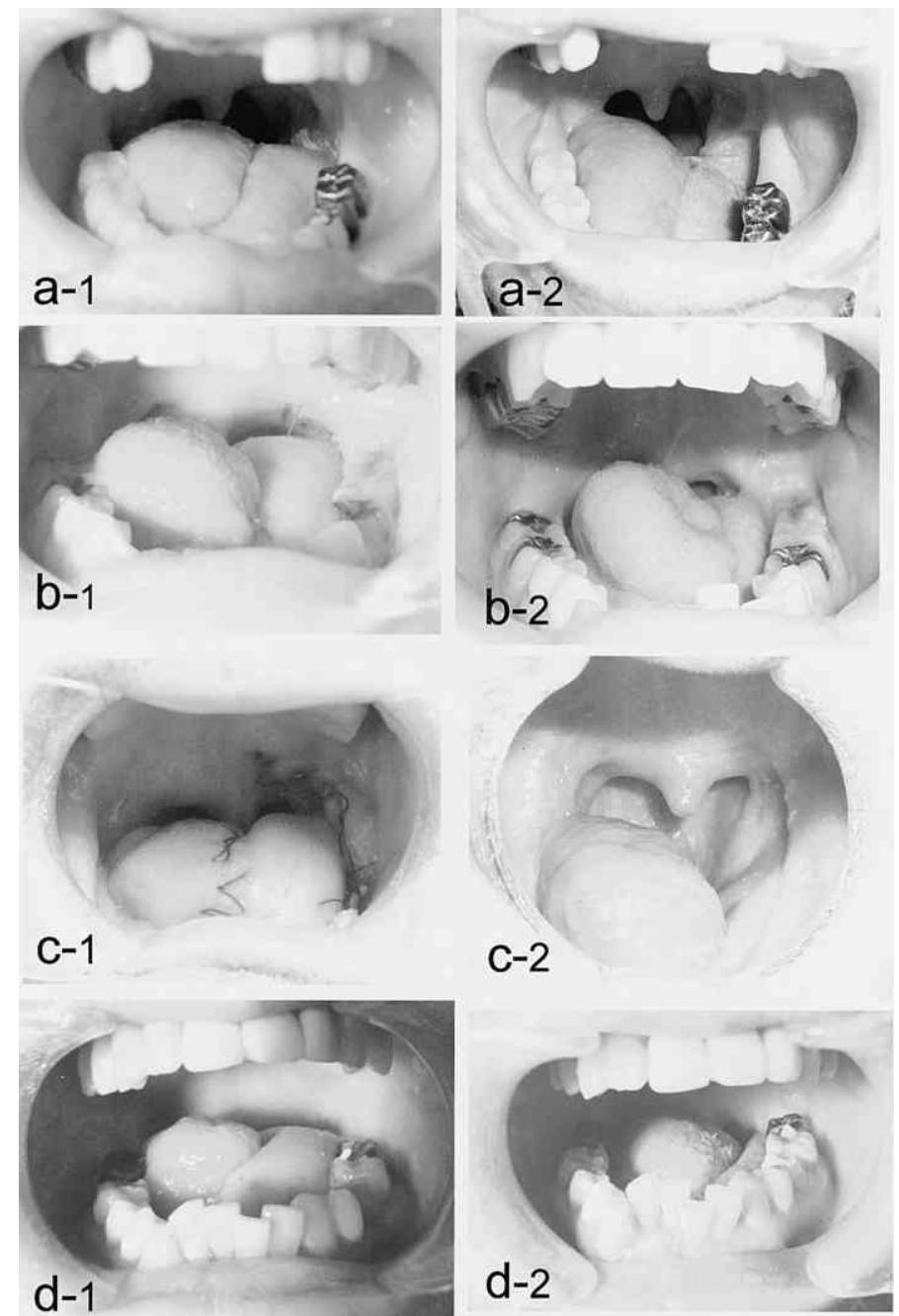

Fig. 2 Serial changes in the flap

$$
\begin{aligned}
& \text { a : Case } 1 \\
& 1: 1 \text { month after surgery } \\
& 2: 12 \text { months after surgery } \\
& c: \text { Case3 } \\
& 1: 1 \text { month after surgery } \\
& 2: 12 \text { months after surgery }
\end{aligned}
$$

\section{b : Case2}

$1: 1$ month after surgery

$2: 12$ months after surgery

d: Case 4

$1: 1$ month after surgery

$2: 12$ months after surgery

\section{Discussion}

There have been a few reports about swallowing function in patients who underwent hemiglossectomy and reconstruction with a pectoralis major myocutaneous flap ${ }^{4-6)}$. However, the evaluation periods ranged from 2-7 months postoperatively and the items evaluated also varied, for instance, functional evaluation, video fluorography and swallowing pressure. In this study, the evaluation period was 12 months postoperatively because we supposed that the flap and swallowing function would have become relatively stable by that time. 

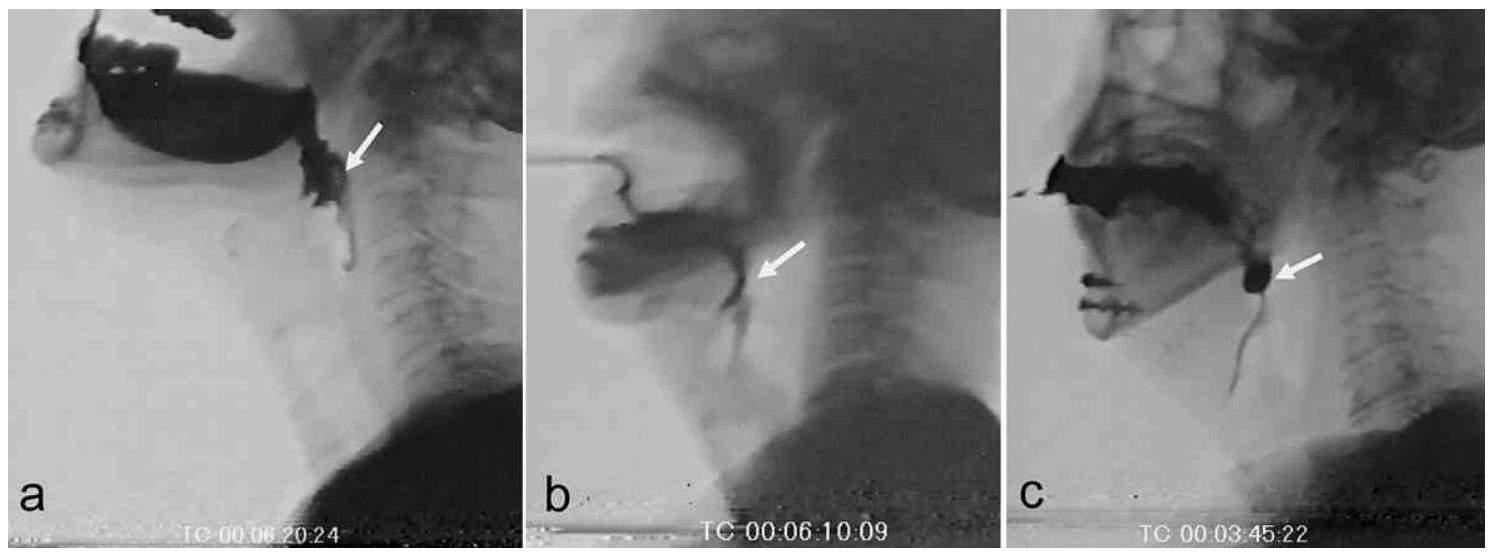

Fig. 3 Holding test diet in the oral cavity

a : Case1 Difficulty (Arrow), b : Case3 Difficulty (Arrow), c : Case4 Difficulty (Arrow)
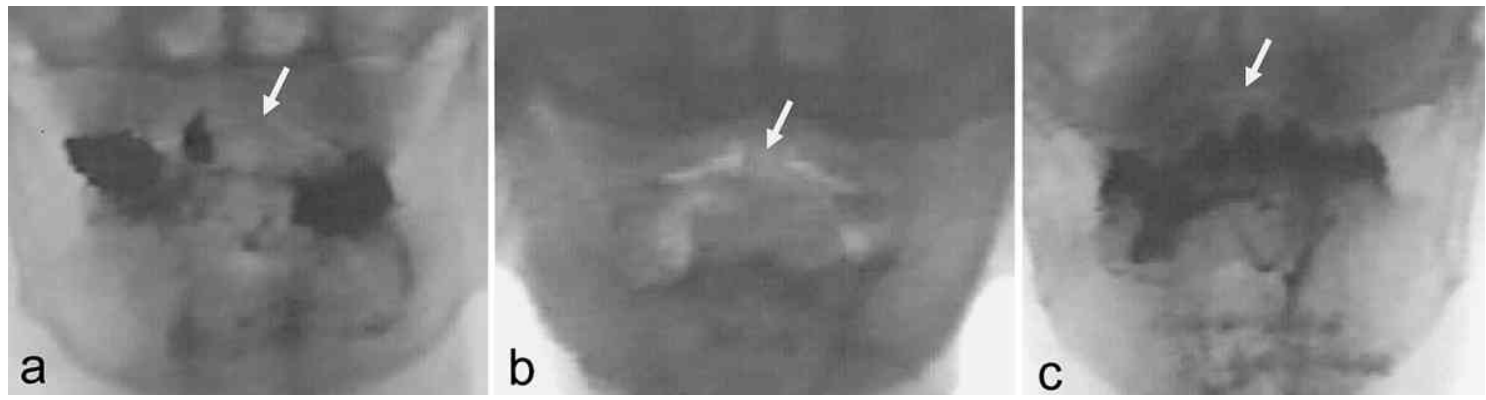

Fig. 4 Tongue movement (Contact between hard plate and tongue)

a : Case1 Possible (Arrow), b : Case3 Possible (Arrow), c : Case4 Possible (Arrow)

Items evaluated were functional evaluation and video fluorography. Particularly, the degree of maximal widening of the esophageal entrance during swallowing was examined using an image analysis system.

1) Serial changes in the flap

Kawaguchi et al suggested that the pectoralis major myocutaneous flap descends downward with shrinkage of the pectoralis major muscle postoperatively ${ }^{4}$. In this study, Flaps in all patients had decreased at 12 months postoperatively in comparison with the size one month postoperatively due to downward descent with shrinkage of the pectoralis major muscle. Particularly, the flap in case 3 decreased remarkably and descended downward. As the reason the flap in case 3 decreased remarkably and descended downward, we considered that case 3 was more slightly built (BW $35.8 \mathrm{~kg}$ • BMI 15.6) in comparison with other cases (case 1 : BW 58. $4 \mathrm{~kg} \cdot \mathrm{BMI} 22.7$, case 2 : BW $49 \mathrm{~kg} \cdot \mathrm{BMI} 17.8$, case 4 :BW $49 \mathrm{~kg} \cdot \mathrm{BMI} 19.1$ )

2) Functional evaluation

Kawaguchi et al suggested that $75 \%$ of patients who underwent hemiglossectomy and reconstruction with a pectoralis major myocutaneous flap could ingest a normal diet, while $25 \%$ of patients could ingest only a soft $\operatorname{diet}^{4)}$. In this study, all patients were capable of oral ingestion and there was no apparent aspiration of water (subjective symptom). Patients who underwent excision of less than $50 \%$ of the radix linguae could consume an approximately normal diet. However, patients who underwent 

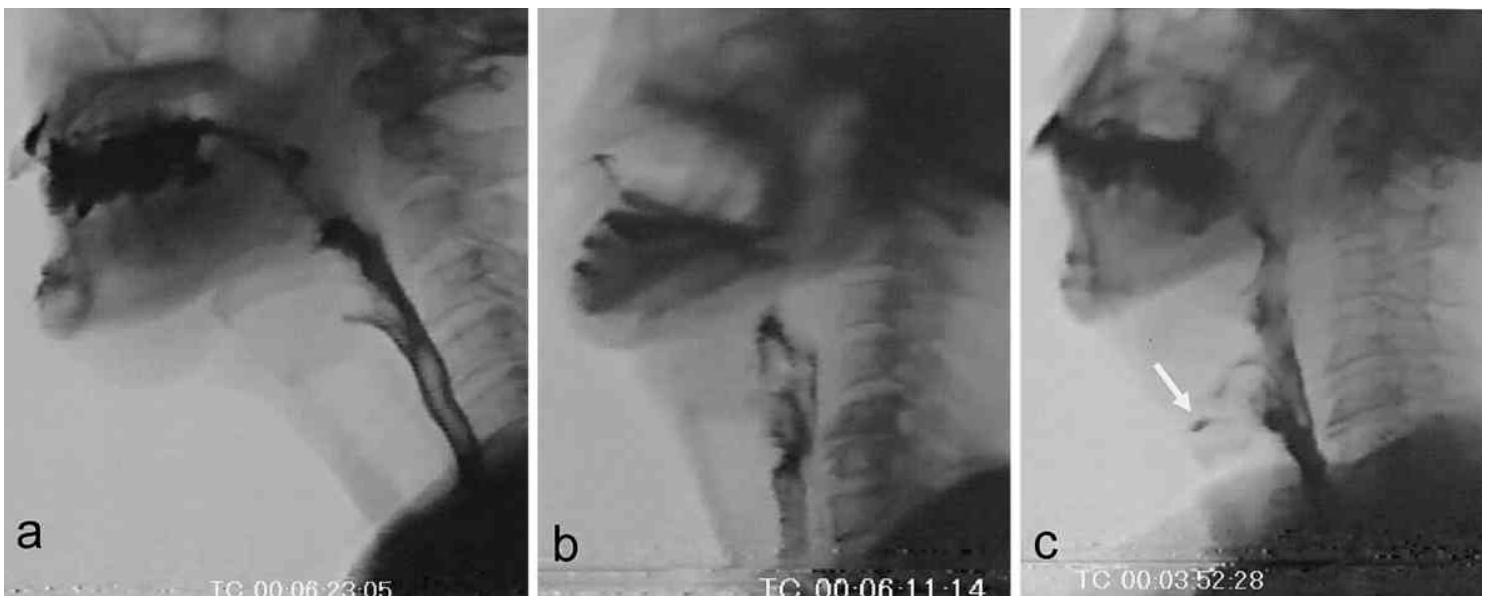

Fig. 5 Penetration / Aspiration

a : Case 1 No findings, b : Case3 No findings, c : Case4 Penetration (Arrow)
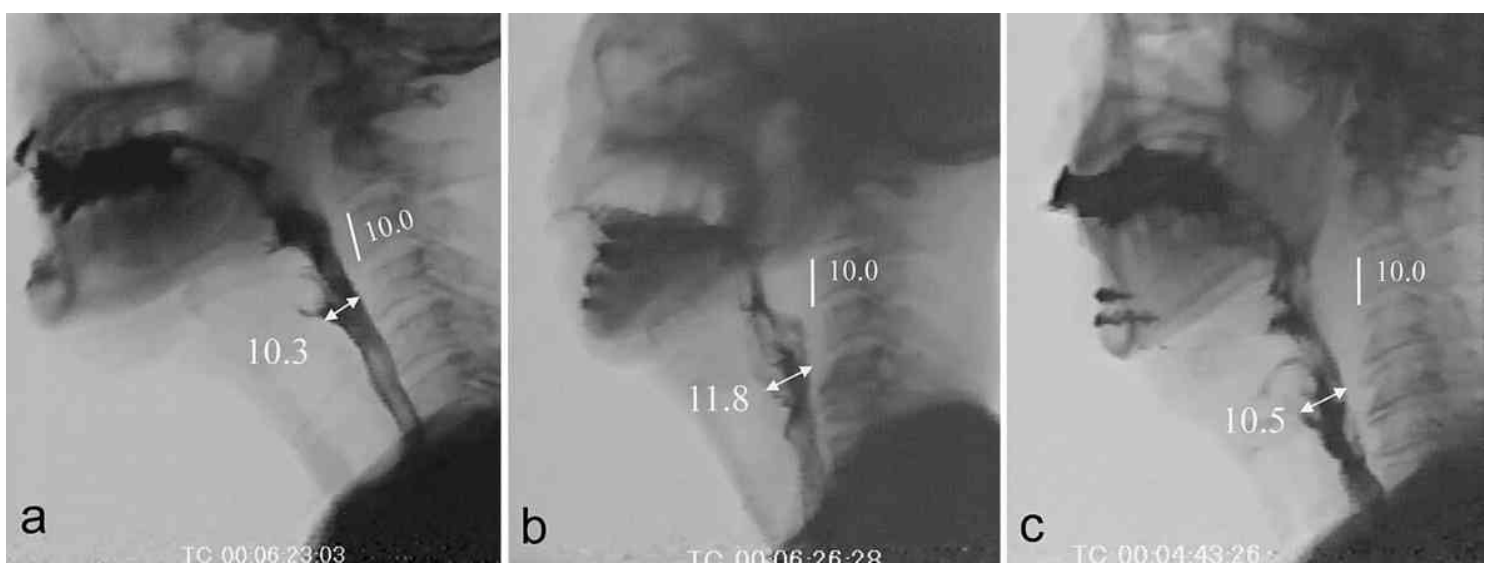

Fig. 6 The degree of maximal widening of the esophageal entrance

a : Case1 Good (Arrow), b : Case3 Good (Arrow), c : Case4 Good (Arrow)

excision of more than $50 \%$ of the radix linguae could required strict limitations of diet. Therefore, it is necessary for patients who undergo excision of more than $50 \%$ of the radix linguae to receive reconstruction with bulky free flaps such as the rectus abdominis flap ${ }^{7}$.

3) Video fluorography

Yamashita et al suggested that mild or moderate aspiration was observed but elevation of the larynx and opening of the esophageal inlet were good in patients who underwent hemiglossectomy and reconstruction with the pectoralis major myocutaneous flap, and suggested that pressures in the hypopharynx and cervical esophagus were almost normal in those patients ${ }^{5}$. We evaluated swallowing function in detail using video fluorography. With regard to holding the test diet in the oral cavity, Hsiao et al suggested that flowing of the test diet into the pharynx was not observed in any patient who underwent hemiglossectomy and reconstruction with the free radial forearm flap ${ }^{8)}$. In this study, none of the patients could hold the test diet in the oral cavity, and flowing of the test diet into the pharynx was observed. we considered that the pectoralis major 
myocutaneous flap had descended downward. Therefore, it was difficult for all patients to hold liquid diet in the oral cavity. With regard to tongue movement, Hsiao et al suggested that patients who underwent hemiglossectomy and reconstruction with the free radial forearm flap could lift the residual tongue and establish good contact between the residual tongue and hard palate $^{8)}$. In this study as well, all patients could shift the residual tongue to the flap side as a compensatory function and the residual tongue could make good contact with the hard palate. Therefore, tongue movement in patients who underwent hemiglossectomy and reconstruction with a pectoralis major myocutaneous flap was comparatively good. With regard to widening of the esophageal entrance during swallowing, we evaluated the degree of maximal widening of the esophageal entrance using an image analysis system. The normal value for the degree of maximal widening of the esophageal entrance ranged from 6.2-11.3 (ave.8.4) relative$1 y^{2}$. In this study, the degree of maximal widening of the esophageal entrance was 10.311.8. Those numerical values indicated nearly normal esophageal widening. Therefore, there was no aspiration in any patient. We suggested that swallowing function in patients who underwent hemiglossectomy and reconstruction with the pectoralis major myocutaneous flap was good approximately 12 months postoperatively. In the future, it will be necessary to examine swallowing function in patients who underwent subtotal resection of the moving element of the tongue or subtotal glossectomy followed by reconstruction using the pectoralis major myocutaneous flap in order to compare swallowing function.

\section{References}

1) Rikimaru $H$ : Three-dimensional anatomical vasucular distributon in pectoralis major myocutaneous flap. J Jpn Kurume Med Assoc 67 : 8-23, 2004

2) Matsunaga K, Oobu K, Ohishi M, et al : The evaluation of swallowing function in unilateral radical neck dissection cases using the picture analysis equipment.-Analysis about the position of the hyoid and opening of the esophageal entrance-. J Jpn Soc Stomatognath Funct 11 : $35-40,2004$

3) Matsunaga K, Nakamura N, Oobu K, et al : A postoperative case of oral cancer in which widening of the esophageal entrance with a balloon catheter enabled oral intake. J Jpn Oral Maxillofac Surg 52 : 524-526, 2006

4) Kawaguchi K, Satou J, Iida N, et al : Assessment of reconstruction following hemiglossectomy of limited mobile tongue. J Jpn Head and Neck Cancer 30 : 105-110, 2004

5) Yamashita H, Kumamoto Y, Kuratomi Y, et al : Swallowing function after hemiglossectomy in patients with tongue cancer. J Jpn Otologia Fukuoka 42 : 456-460, 1996

6) Watanabe H, Shin T, Matsuo K, et al : The pectoralis major myocutaneous flap for reconstruction. J Jpn Otologia Fukuoka 39 : 319-325, 1993

7) Matsunaga K, Oobu K, Ohishi M : A clinical study on pre-and postoperative swallowing function in tongue cancer patients. JJDR $6: 53-$ 64, 2002

8) Hsiao HT, Leu YS, Chang SH, et al : Swallowing function in patients who underwent hemiglossectomy: comparison of primary closure and free radical forearm flap reconstruction with videofluoroscopy. Ann Plast Surg 50 : 450-455, 2003 